\title{
Grinding Process Validation Approach (gPVA)
}

\author{
C. Vogt $^{1}$, O. Faehnle ${ }^{2}$ and R. Rascher ${ }^{1}$ \\ 1. IPH Department, Deggendorf Institute of Technology, Deggendorf 94469, Germany \\ 2. Fisba AG, St. Gallen 9016, Switzerland
}

\begin{abstract}
This paper presents a load-controlled gPVA (grinding Process Validation Approach) for setting up feed-controlled CNC (Computer Numerical Control) grinding processes for the generation of optical elements. The gPVA enables the quantitative determination of process windows for CNC grinding processes as well as the identification of optimized sets of process parameters without the need to run tests on actual production parts in the industrial workshop occupying expensive CNC machining time.
\end{abstract}

Key words: Grinding, process optimization, productivity enhancement.

\section{Introduction}

Production processes must be stable and predictable to enable the determination of the optimum fabrication time and yield, resulting in the minimum fabrication cost and machine capacity required.

During the generation of optical elements, polishing is the most time-consuming process along the manufacturing chain. Besides the need to apply the optimum set of polishing process parameters, polishing time strongly depends on the initial characteristics of the workpiece surface which have been generated in the previous grinding steps. Furthermore, grinding is also applied at the end of the manufacturing chain for the centering of lenses or cemented optical elements. Therefore, grinding is among the most important and risky processes in optical fabrication.

Consequently, an optimized sequence of grinding processes, ranging from rough shaping to fine grinding, has to be established, each process having an optimum set of FPs (fabrication parameters) to be controlled during serial production by FPC (fabrication parameter controlling). In order to identify the optimum set of FPs for each grinding process, its process window needs to be determined

Corresponding author: C. Vogt, graduate engineer, research field: grinding of optical components. depending (among others) on the applied tool, the workpiece material characteristics and the workpiece shape.

To achieve that aim, quality improvement methods are employed, such as the three wagon methodology [1], which has been developed especially for the optimization of optical production chains. It is focused on the process rather than on machine optimization and essentially comprises three subsequent project phases: (wagon 1) freeze starting situation (identify and freeze critical FPs, determine their flow chart and install FPC); (wagon 2) optimize the set of FPs (one at a time); and (wagon 3) verify the optimized set of FPs (by producing test batches, monitoring yield and fabrication cost).

Unfortunately, a major problem with the design of grinding processes is the lack of performance data for commercially available grinding tools. For this reason, grinding operations are usually set up experimentally by testing different FP sets on actual workpieces, which render the whole optimization process risky, cost intensive and ineffective.

This paper presents a load-controlled approach, hereinafter referred to as the gPVA (grinding Process Validation Approach), both to determine the process window for $\mathrm{CNC}$ grinding processes and to set up optimized sets of FPs without the need to run tests on actual production parts in the industrial workshop. 


\section{2. gPVA}

From Preston [2],we know that, within the process window of load-controlled abrasive grinding processes, for a fixed relative speed $\mathrm{v}_{\mathrm{c}}$ between the tool and the workpiece surface, there is a linear dependency between the applied tool pressure $\mathrm{P}$ and the workpiece wear MRR (material removal rate) generated thereby. The inclination of MRR is determined by the so-called Preston coefficient or tool index (see Eq. (1)).

$$
\begin{aligned}
& M R R=\left(k * v_{c}\right)^{*} \mathrm{P} \\
& \mathrm{MRR}=\text { Material Removal Rate }[\mathrm{mm} / \mathrm{sec}] ; \\
& \mathrm{k}=\text { Tool Index }\left[\mathrm{mm}^{2} / \mathrm{N}\right] ; \\
& \mathrm{P}=\text { Pressure }\left[\mathrm{N} / \mathrm{mm}^{2}\right] ; \\
& \mathrm{v}_{\mathrm{c}}=\text { Cutting Speed }[\mathrm{mm} / \mathrm{sec}] .
\end{aligned}
$$

The argumentum and contrario implies that outside the process window there is no linear dependency of the tool pressure on workpiece wear. In this case, the applied machining energy is not transferred purely to workpiece wear but additionally causes other unwanted effects such as tool wear, heat, as well as mechanical stress within the machine. This affects yield and machining time and consequently fabrication cost and machine capacity.

In the authors' perception, this can be applied to determine process windows for $\mathrm{CNC}$ grinding processes as well as to optimize their set of FPs. Even the most rigid $\mathrm{CNC}$ grinding machines employ spindles, drives and engines of finite stiffness. Consequently, feed-controlled movements within CNC machines are determined by load-controlled processes of the highest rigidity.

To that aim, (gPVA) the following three-step procedure is carried out within the gPVA:

gPVA:

(a) Freezing boundary conditions;

(b) Experimental detection of grinding process window; and

(c) Determination of optimum set of FPs.

(1) Freezing boundary conditions. Setting up boundary conditions by freezing the coolant type and cutting speed (for a given tool and workpiece material) and nulling the tool surface are by dressing.

- Freeze the coolant. The grinding coolant has a major effect on the chipping and wearing behavior during grinding. Most workshops use one type of coolant for all grinding operations. Larger workshops often use a central supply, so that the type of coolant must be taken as a given parameter that cannot be changed depending on individual needs.

- Freeze the cutting speed $\mathrm{v}_{\mathrm{c}}$. Due to productivity issues, there is a tendency to set the cutting speed as high as possible. The available range of cutting speeds depends on the machinery used. Typical values are within the $10-30 \mathrm{~m} / \mathrm{sec}$ range for fine grinding processes. Applying the gPVA, three $\mathrm{v}_{\mathrm{c}}$ values are frozen and tested successively. As a starting point, an average value of e.g. $20 \mathrm{~m} / \mathrm{sec}$ is fixed followed by two other values, one located at the upper end of the $\mathrm{v}_{\mathrm{c}}$ range and one at the lower. In addition, checking the range of spindle and tool resonance frequencies should be considered in order to avoid chatter and unwanted mid-spatial frequencies.

- Freeze the initial tool surface. During initial dressing, a good radial run-out minimizes vibrations and assures that the set parameters according to infeed and depth of cut are applied as planned. In addition to that, the dressing operation removes the adherence material from former grinding operations and assures that dull abrasives are absent at the start of processing. Dressing operations can be carried out using sharpening stones or eroding techniques for electrical conductive bond materials.

Applying the gPVA, it is important that the boundary conditions should be kept constant to eliminate their possible influence on the parameter setting.

(2) Detection of grinding process window. With the boundary conditions set, the process window for a $\mathrm{CNC}$ grinding tool and the workpiece material is determined by using the gPVA outside the industrial fabrication department using a CNC machine 
equipped with a testing device for tool pressure.

The gPVA rests on the fact that, for a fixed cutting speed $v_{c}$, there is a range where the tool pressure and the MRR are approximately proportional to each other. This range can be determined using a CNC machine, where the MRR is applied while the pressure within the area of contact between the tool and the work piece is recorded [3]. The test rig utilized for this approach consists of the "to be tested" grinding tool, a sample of the "to be machined" work piece material and a dynamometer (see Fig. 1).

The pressure and the material removal rate being proportional allow the use of Preston's equation to describe the dependency of input parameters and measured output values within the process window as Eq. (2):

$$
M R R=\frac{A_{\text {feed }} * v_{\text {feed }}}{A_{\text {contact }}}=\left(k * v_{c}\right) * \mathrm{P}
$$

$\mathrm{A}_{\text {feed }}=$ Size of the tool and workpiece contact area, perpenticular to the feed direction;

$\mathrm{v}_{\text {feed }}=$ Feed speed;

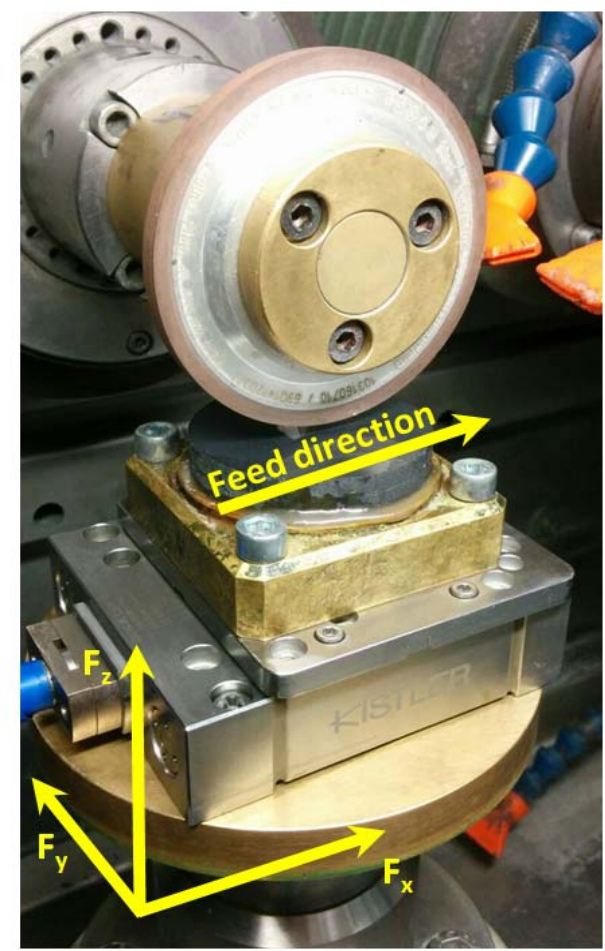

Fig. 1 Experimental gPVA setup to determine the gPVA curve: $\operatorname{MRR}(P)$.
$\mathrm{A}_{\text {contact }}=$ Total size of the area of contact between the tool and the workpiece.

Fig. 2 shows the results of a typical gPVA experiment. Each feed speed generates an average force level. The assignment for the given example can be found in Fig. 2 left. As the graph shows, the force rises in proportion to the feed speed until $\mathrm{v}_{\text {feed }}=645$ $\mathrm{mm} / \mathrm{min}$. Above this level, the force grows exponentially. Turning this into a correlation of pressure and the removal rate, then it is obvious that a further increase of pressure does not necessarily lead to a rise in the MRR (Fig. 2 right). Beyond this point, the supplied energy tends to generate, among others, more tool wear, which leads to a higher probability of stress marks, structures and geometrical irregularities. This part of the curve is marked as the "Overload" area.

In order to compare the gPVA curves recorded at different cutting speeds $\mathrm{v}_{\mathrm{c}}$, their gradients (see Eq. (2)) have to be converted to the cutting speed of $v_{c}=$ $1 \mathrm{~m} / \mathrm{sec}$.

Fig. 3 shows a typical example for determining a process window for a fixed cutting speed and coolant. For increasing values of tool pressure the generated workpiece wear is measured. The limit of the process window has been reached when the linear dependency between tool pressure and workpiece wear ends.

The gPVA curves and the size of the process window can be analyzed more easily having an additional look at the second derivative of $M R R(P)$ (see Eq. (3) and Fig. 4):

$$
\operatorname{MRR}(P)^{\prime \prime}=\frac{\partial^{2} M R R}{(\partial P)^{2}}
$$

A change in the curve's gradient of more than $100 \%$ of its value (see Fig. 4) is an indicator that the process has become unstable determining the maximum pressure at the upper edge of the process window, where the linear dependency of MRR on $P$ ends. For the example shown in Figs. 3 and 4, this is the case at around $\mathrm{P}=5 \mathrm{~N} / \mathrm{mm}^{2}$. 


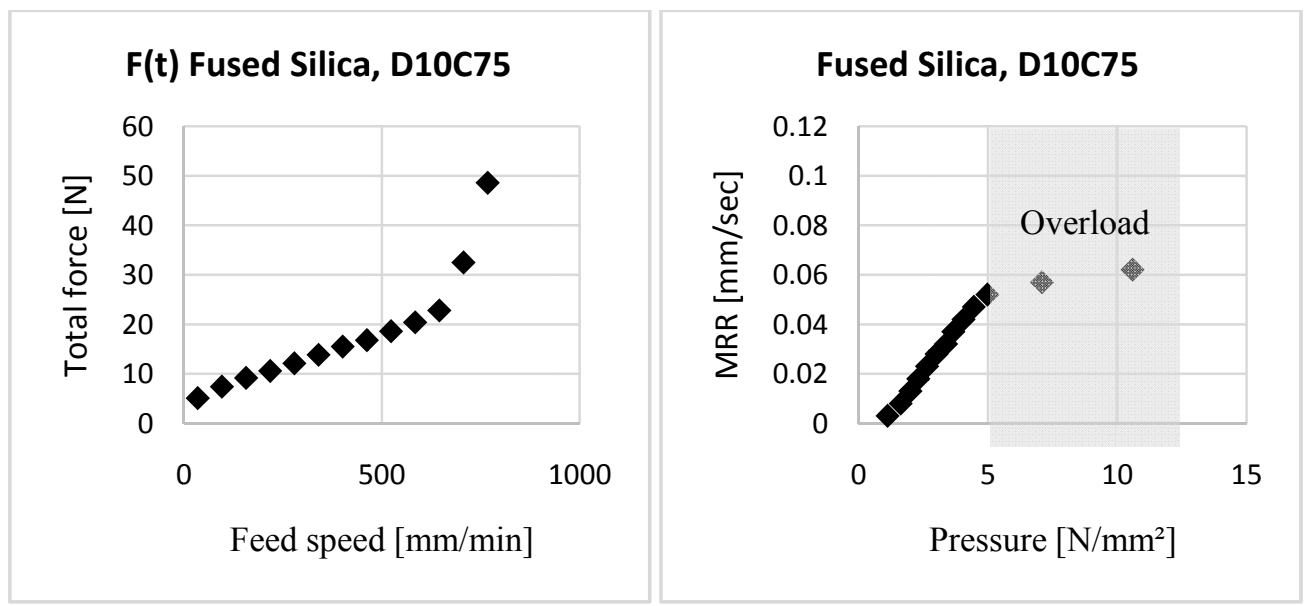

Fig. 2 Force versus feed speed (left) and gPVA curve $(\operatorname{MRR}(P))$ (right) for the D10C75 metal bond, tested at $\mathrm{v}_{\mathrm{c}}=16 \mathrm{~m} / \mathrm{sec}$.

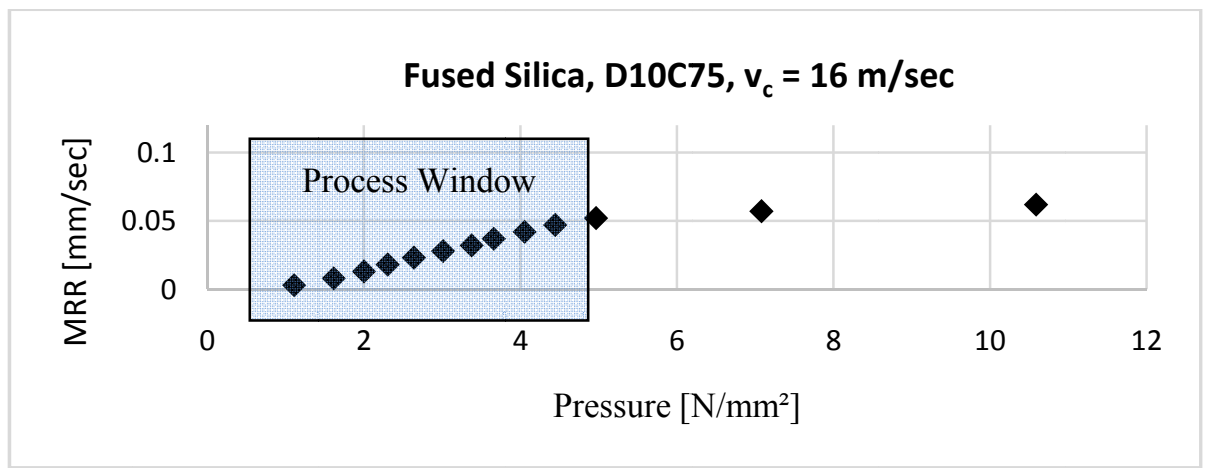

Fig. 3 Typical gPVA curve showing the process window for a CNC grinding process.

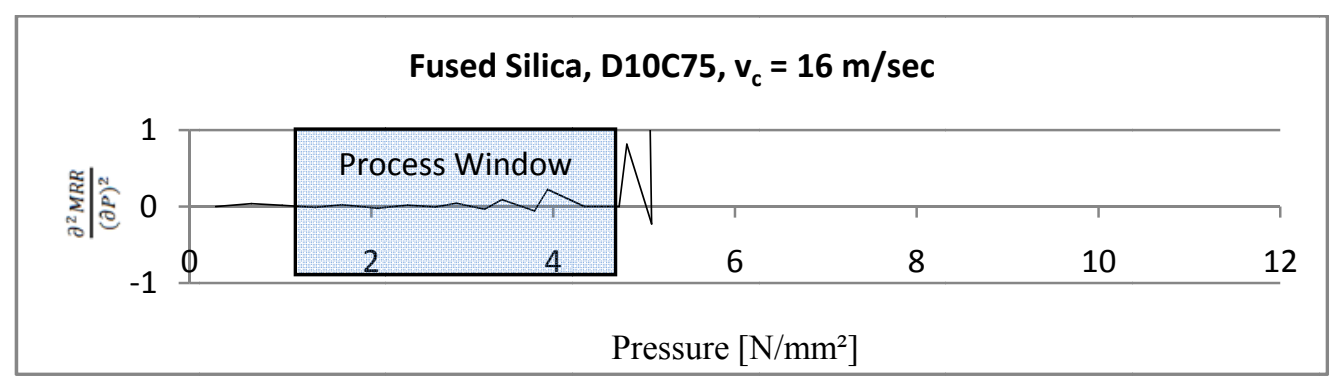

Fig. 4 Second derivative of $\operatorname{MRR}(P)$ for a typical gPVA curve indicating the upper limit of the process window shown in Fig. 3.

(3) Determination of optimum set of FPs. With the gPVA characteristic curve and the process window determined (see Fig. 3 and 4), the optimum working point and its set of FPs can be determined by using the formula given in Eq. (2). The size of the area of contact and the removed amount of material per time depends on the tooling strategy (e.g. cup wheel applied with line contact or disc wheel applied in single point contact mode), tool dimensions as well as the depth of cut and tool infeed. The optimum working point along the gPVA curve should be chosen at high MRR values, with a sufficient safety distance to the upper process window limit.

\section{Accuracy Considerations}

The applied load is the most critical influence factor of all. The maximum possible load decreases at higher cutting speeds and poor cooling conditions.

Fig. 5 presents an error bar chart for a D15C50 brass bond grinding tool to give an insight about 
gPVA method accuracy. The experimental dataset shown was generated by three runs under identical conditions. The tool was not re-dressed after the single runs.

The size of the error bar yield is $2 \mathrm{x} \sigma$, which means that $95 \%$ of all measurements will be within the range of the given bars for typical curves. This leads to an uncertainty of $\pm 112 \%$ for pressures around $0.8 \mathrm{~N} / \mathrm{mm}^{2}$ and $\pm 12 \%$ around $4 \mathrm{~N} / \mathrm{mm}^{2}$.

\section{Influencing Factors}

In what follows, the influence of different cutting speeds, types of the coolant and workpiece materials on gPVA curves are analyzed.

Cutting Speed $\mathrm{v}_{\mathrm{c}}$ : For a given tool and workpiece material, different cutting speeds and their influence on the gPVA curves were tested to cover commonly used parameters in manufacturing precision optics. The results are presented in Fig. 6, where the gPVA curves within the process window are shown. The perpendicular line at the end of each curve represents the uncertainty range for each measurement (see also Fig. 5).

Higher cutting speeds cause enhanced force and pressure levels for the same MRR. On the other hand, the maximum achievable pressure decreases at higher cutting speeds. The maximum pressure for $\mathrm{v}_{\mathrm{c}} 13 \mathrm{~m} / \mathrm{sec}$ was $4.8 \mathrm{~N} / \mathrm{mm}^{2}$, while the highest valid pressure for $\mathrm{v}_{\mathrm{c}}$ $30 \mathrm{~m} / \mathrm{sec}$ was $3.6 \mathrm{~N} / \mathrm{mm}^{2}$ (see Fig. 6). The gPVA curves show a clear decrease of the maximum possible MRR at higher cutting speeds accompanied by a generally higher pressure level.

Coolant: For a given tool and cutting speed, the coolant has a major impact on the grinding process. The influence of commercially available, water-soluble lubricants was tested with one coolant especially developed for grinding glass, hereinafter called coolant A. Another coolant, coolant B, was developed for milling and grinding applications. For this reason, it is used at higher concentrations. The

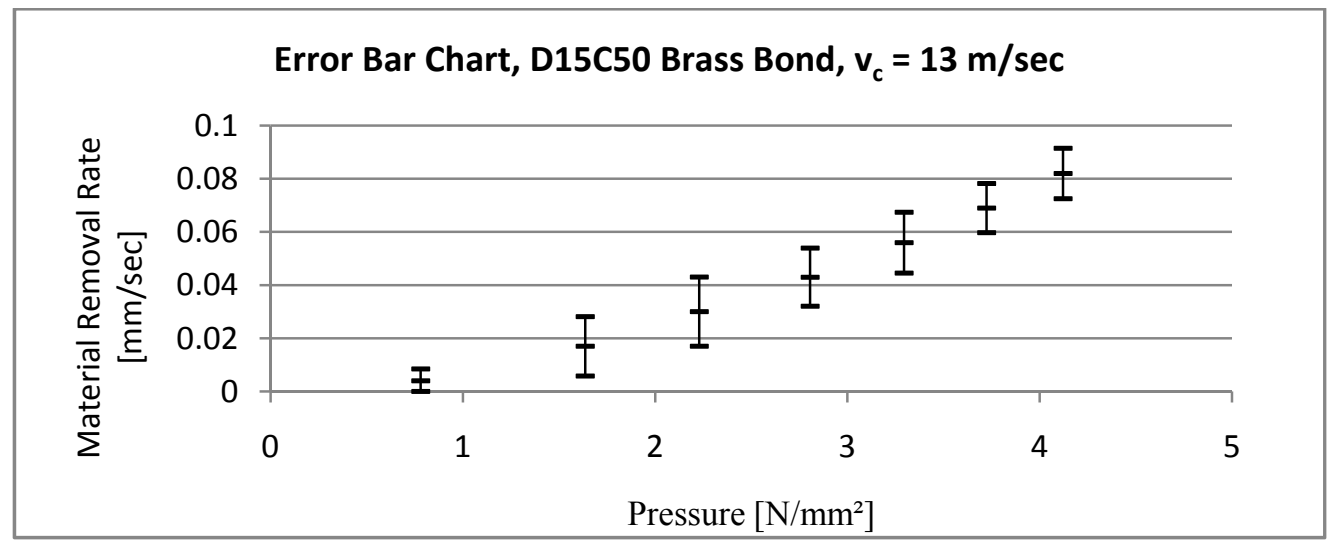

Fig. 5 Error bar chart for typical gPVA curves (D15C50 brass bond on fused silica).

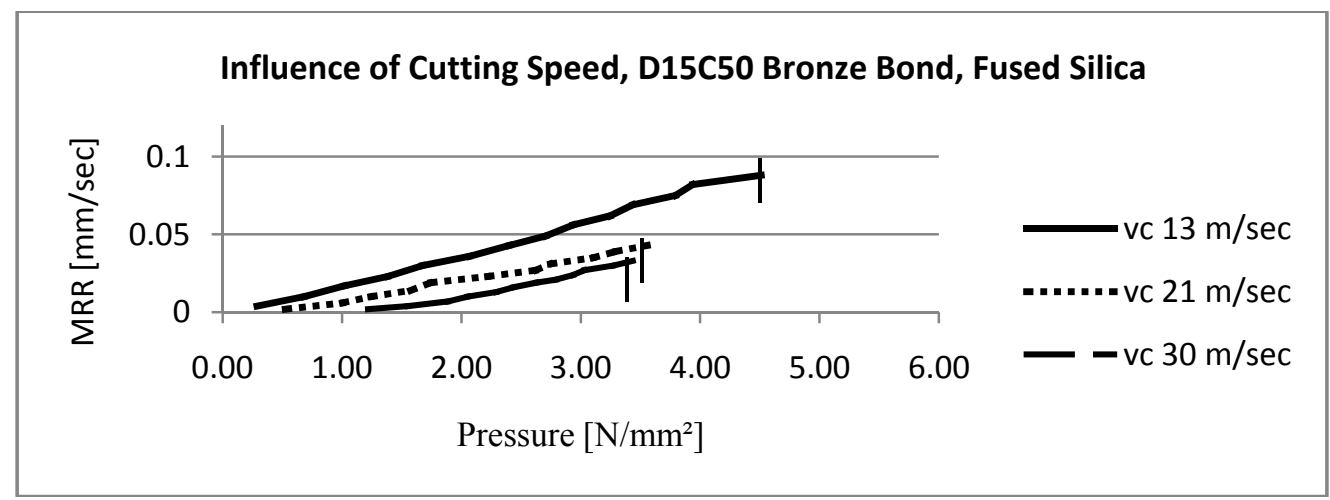

Fig. 6 gPVA curves for fused silica applying three different cutting speeds $v_{c}$. 
graph given in Fig. 7 shows the performance test results for coolants $\mathrm{A}$ and $\mathrm{B}$. The MRR reaches its limit at a pressure of $0.9 \mathrm{~N} / \mathrm{mm}^{2}$ for coolant $\mathrm{B}$, which indicates increased tool wear, whereas coolant $\mathrm{A}$ reaches the limit at $1.8 \mathrm{~N} / \mathrm{mm}^{2}$, resembling a $200 \%$ higher maximum MRR compared to coolant A.

Material: As expected, for a given tool and cutting speed and coolant, the material has a massive impact on the process characteristics and the achievable stock removal. Experiments on SF57 and fused silica led to the gPVA curves shown in Fig. 8. SF57 has a lower hardness (HK 350) and grinding ability class (HG 1, according to ISO 12844) compared to fused silica (HK 570 and HG 2).

The maximum possible MRR was measured to be $0.05 \mathrm{~mm} / \mathrm{sec}$ for fused silica, while the MRR was limited to $0.018 \mathrm{~mm} / \mathrm{sec}$ for SF57, which is about a third.

\section{Discussion}

The results show that different major influencing factors for the gPVA method must be taken into account for designing efficient grinding processes. Some of them must be considered at given circumstances, such as coolant, workpiece material and type of grinding tool, while the cutting speed has to be set within the first step of the three-step gPVA method to enable the recording of the gPVA curve. The experimental results point out that the presented method can be used to design grinding processes off-line to determine suitable parameters.

While the traditional trial and error approach leaves unused productivity potential, the process limits using the gPVA are quantitatively determined and the process parameters can be set to achieve the highest possible MRR within the process window. In doing so,

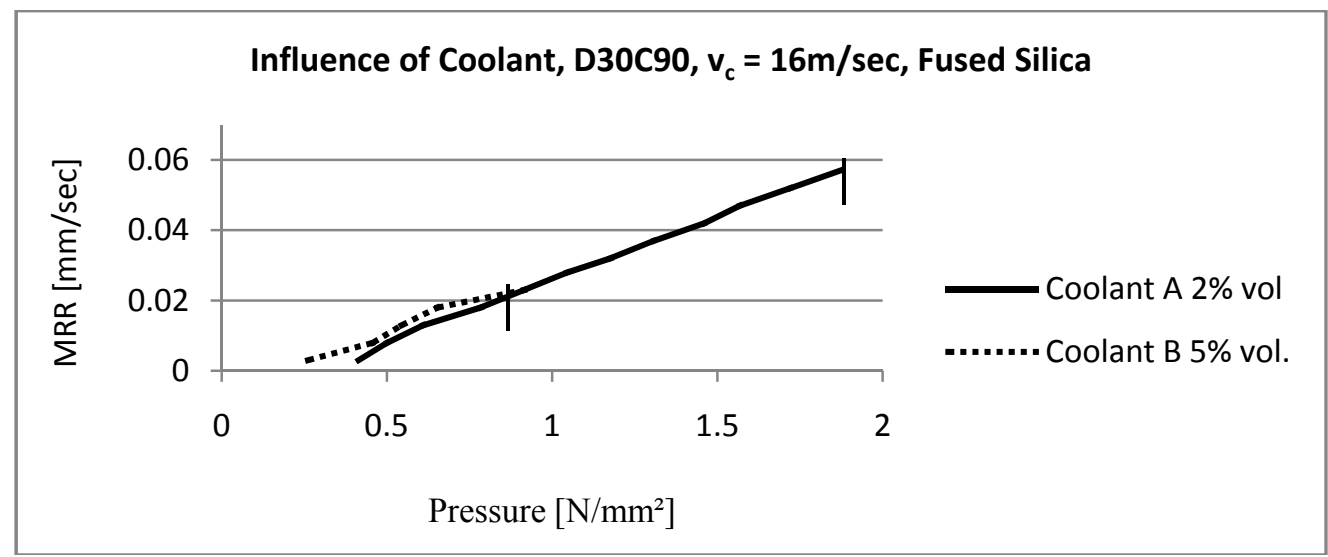

Fig. 7 gPVA curves for two different coolants on fused silica.

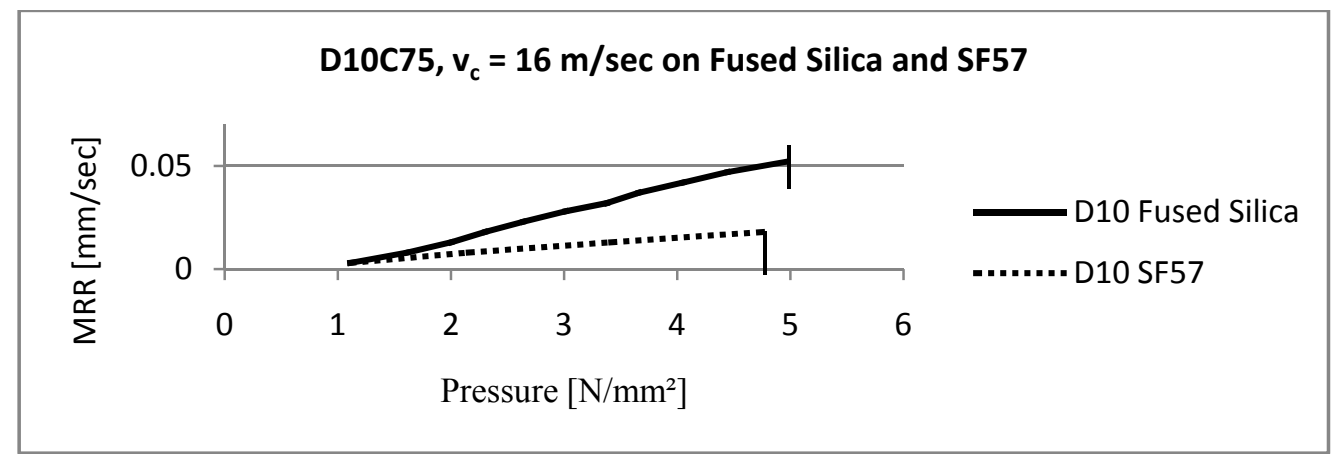

Fig. 8 gPVA curve of a D10C75 tool applied to two materials: SF57 and fused silica. 


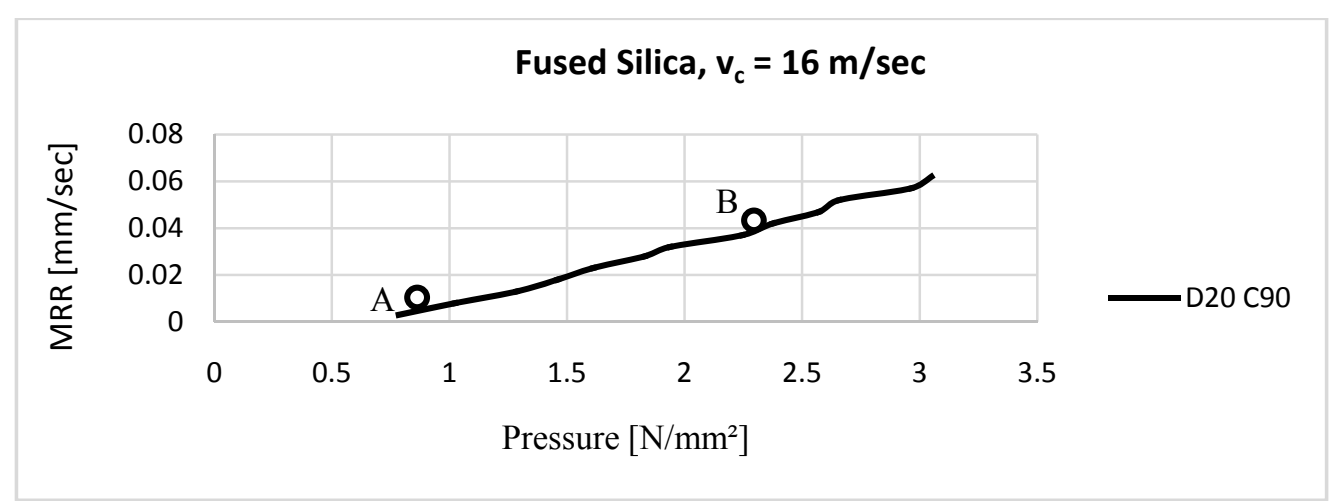

Fig. 9 gPVA curve of a D20C90 tool applied to fused silica, industrial case study.

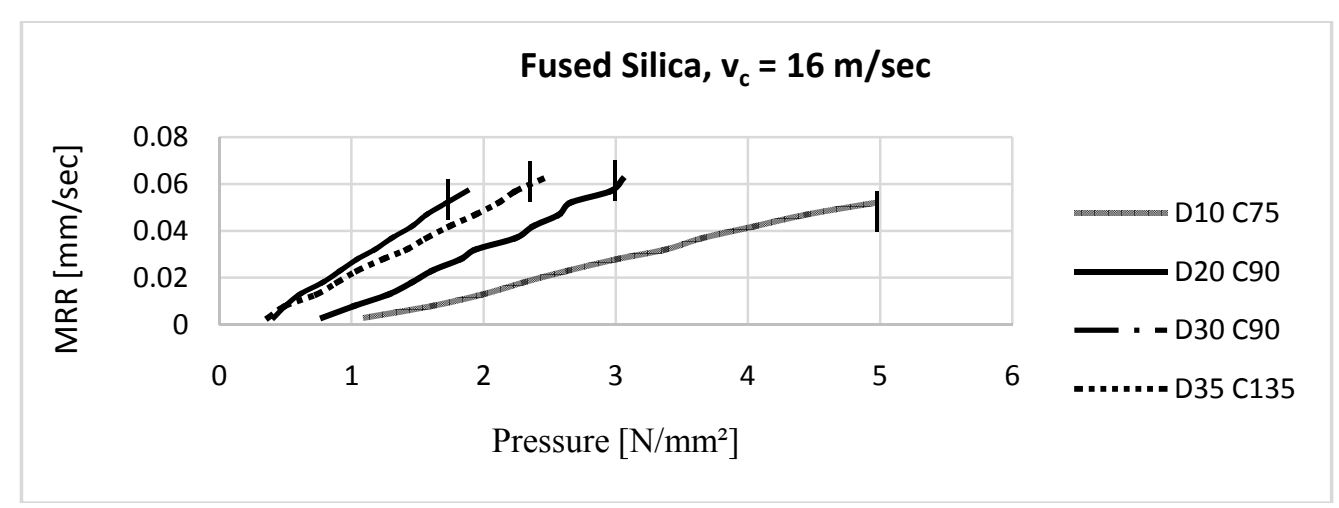

Fig. 10 gPVA curves for different grinding tools for fused silica.

it is recommended to leave some space to the upper process window border for unexpected events, such as poor cooling conditions or a bad radial run-out of the grinding tool.

As an example, Fig. 9 shows the application of the gPVA method at an industrial partner to determine the grinding process window and optimized set of FPs for an existing grinding tool [4]. In this case, the initial situation installed previously by traditional trial and error optimizations is indicated by point A. After the determination of the process window, the set of FPs could be optimized without using actual production parts in the department. The optimized working point is indicated by point B improving yield from 70 to $98 \%$ and reducing production time by $12 \%$ at a batch size of 100 lenses.

In addition, the gPVA can be applied for setting up an optimized sequence of grinding steps along a fabrication chain, ranging from rough to fine grinding. Fig. 10 shows the performance characteristics of our different tools, featuring different grain sizes on fused silica as an example. A classic tooling sequence would be D35, D20 and D10. As the curves show, the peak MRR values of D35, D30 and D20 are in this case about the same. So a smarter sequence would be to start directly with D20 followed by D10. That way a whole machining step can be skipped, leading to shorter fabrication times.

\section{Conclusions}

This paper presents a load-controlled approach, gPVA, to determine both the process window for feed-controlled $\mathrm{CNC}$ grinding processes as well as to generate optimized sets of FPs without the need to run tests on actual production parts in the industrial workshop occupying expensive $\mathrm{CNC}$ machining time. A methodology for the systematic design of grinding processes using the gPVA has been presented. It was demonstrated that the systematic generation of tool performance maps for defined materials enables optimized process design. Doing so, optimum points of operation for individual tools can be identified 
saving fabrication cost. In addition to that, different tools can be compared to each other in terms of removal performance and the generated force levels. This enables the selection of the best possible tooling sequence reducing manufacturing times.

In conclusion, the gPVA reveals a high potential in setting up grinding processes of high stability and productivity that can hardly be determined using the more traditional "trial and error" approach.

Currently, the gPVA is applied at the Deggendorf Institute of Technology to generate comparable and standardized performance data for commercially available grinding tools from different vendors by testing them in a standardized situation with a frozen cutting speed on BK7 glass.

\section{References}

[1] Faehnle, O. 2016. "Process Optimization in Optical Fabrication." Opt. Eng. 55(3)

[2] Preston, F.W. 1918. "The Theory and Design of Plate Glass Polishing Machines." Journal of the Society of Glass Technology 214.

[3] Vogt, C., Sinzinger, S., Rohrbacher, M., and Rascher, R. 2016. "Prediction of Grinding Tool Wear and Lifetime by Using a Test Bench." Proc. SPIE10009, Third European Seminar on Precision Optics Manufacturing, 100090Z.

[4] Vogt, C., Faehnle, O., and Rascher, R. 2017. "Three Wagons Approach Applied to Optimization of Industrial Grinding Processes." OSA Optical Fabrication and Testing Conference OF\&T, Denver, CA, USA. 